
and cancer research in one go

\author{
Fifth International Workshop on Arylamine $\mathbf{N}$-Acetyltransferases \\ University Paris Diderot, Paris, France, 1-3 September 2010
}

Arylamine $\mathrm{N}$-acetyltransferases (NATs) are phase II xenobiotic metabolizing enzymes playing a key role in the detoxification and metabolic activation of aromatic amine xenobiotics. The triennial International NAT Workshop has been an important academic meeting where developments in the study of NATs and aromatic amine metabolism have been presented. The 2010 Workshop took place in University Paris Diderot Paris, France. Topics included: structures and functions of eukaryotic and prokaryotic NATs, gene regulation and expression of human NATs, polymorphisms and their effects, arylamine metabolism and toxicity. Nomenclature issues were also discussed.

\section{Conference details}

This Conference Scene provides an overview of the Fifth International Workshop on Arylamine $N$-Acetyltransferases, which was held at the University Paris Diderot Campus in Paris, France on the 1-3 September 2010. The subjects covered included: molecular and cellular biology; genetics; structural biology; and biochemistry, as well as studies on microorganism models. Presentations were divided into five sessions that are detailed below. A lively discussion concerning NAT nomenclature was held by the NAT Gene Nomenclature Committee [101]. Selected posters were also presented on NAT-related subjects.

\section{NAT polymorphisms, diseases} \& evolution/NAT nomenclature Professor José AG Agundez (University of Extremadura, Badajoz, Spain) reported the identification of copy number variations in human NAT1 and NAT2 genes. Individuals with a single $N A T 2$ copy and individuals with three copies of NAT2 in a population of 361 individuals of European descent were identified. Professor David W Hein (University of Kentucky, KY, USA) presented an international study conducted on a single common tag SNP (rs1495741), located at the 3' end of human NAT2 and associated with urinary bladder cancer risk. This NAT2 tag SNP identified in a genomewide scan, predicts the seven SNP inferred NAT2 phenotype (slow) with high accuracy, and thus can be used as a sole marker in pharmacogenetic and epidemiological studies in populations from European descent. Dr Audrey Sabbagh (Inserm
U745, Paris, France) and Dr Etienne Patin (Pasteur Institute, Paris, France) presented evolutionary pharmacogenetics of the NAT family demonstrating that positive selection of NAT2 haplotypes confers a slow acetylator phenotype, as a possible consequence of changes in modes of subsistence and lifestyles in human populations such as the transition to farming.

Professor Philippe Silar (University Paris-Sud, Orsay, France) talked about the identification and evolution of NAT genes in fungi. Certain fungi possess several NAT genes, whereas others are devoid of it. Dr Sotiria Boukouvala (Democritus University of Thrace, Alexandroupolis, Greece) presented a comparative genomic survey of NAT homologous sequences in 2445 genomes (1532 species). The first examples of $N A T$ homologues in protists, archea and in a giant virus were shown. Eleni P Karagianni from Dr Boukouvala's laboratory reported the cloning and characterization of NAT genes from some mycotoxigenic fungi, such as Fusarium verticillioides M3125 and Fusarium graminearum NRRL 31084 strains.

\section{NAT \& aromatic amine metabolism/animal models}

Professor David W Hein presented data on the associations between human NAT2 SNPs and haplotypes with catalytic activity in cryopreserved human hepatocytes. A strong agreement between the rs1495741 NAT2 tag SNP and the seven SNP inferred NAT2 phenotype was found, and indicated that this tag SNP could be used as a sole marker in pharmacogenetic studies.

\section{Fernando Rodrigues-Lima ${ }^{+1}$,} Julien Dairou ${ }^{1}$, Nicola Laurieri ${ }^{2}$, Florent Busi ${ }^{1}$ \& Jean-Marie Dupret ${ }^{1}$

${ }^{1}$ University Paris Diderot, Sorbonne Paris Cité, Unité BFA, EAC-CNRS 4413, 75013, Paris, France

${ }^{2}$ Oxford University, Department of Pharmacology, Oxford, UK

${ }^{+}$Author for correspondence:

fernando.rodrigues-lima@univ-paris-diderot.fr 
Dr Peter J O'Brien (University of Toronto, ON, Canada) reported results on the increased hepatotoxicity of 4-aminobiphenyl (4-ABP) under oxidative stress conditions. Professor Denis M Grant (University of Toronto) demonstrated that male mice lacking both NAT1 and NAT2 were partially protected from 4-ABP-induced liver tumors. The level of DNA damages could not predict the rate of DNA mutations resulting in tumor initiation. Interestingly, Dr Mark Doll from Professor David Hein's laboratory gave a talk on how differences between human slow NAT2 alleles have an impact on the levels of 4-ABP-induced DNA adducts. He reported that heterogeneity with the 'slow' NAT2 acetylator phenotypes should be taken into account. Carmine S Leggett from Professor David Hein's laboratory presented the use of SV40-transformed human fibroblasts (GM4429) in order to examine the effects of human NAT2 haplotypes in the presence of NAT1 in a NER-deficient human cell.

Professor Charlene McQueen (Auburn University, AL, USA) reported that mice lacking peroxisome proliferator-activated $\alpha$ have greater hepatotoxicity than wild-type mice when exposed to hydrazine, a catabolite of the antitubercular drug isoniazid (metabolized by human NAT2).

In a keynote lecture, Professor Robert Barouki (Université Paris Descartes) presented new results on the storage of persistent organic pollutants (POPs) by adipose tissue.

\section{NAT expression \& regulation}

Professor Rodney Minchin (University of Queensland, Brisbane, Australia) demonstrated that the knockdown of NAT1 in colon carcinoma cells using shRNA drives a marked change in cell morphology, cell contact growth inhibition and viability at confluence. Knocking down NAT1 inhibited invasion and metastasis of breast cancer cells suggesting that NAT1 could play a role in tumorigenesis [1].

Professor Brunhilde Blömeke (Trier University, Trier, Germany) demonstrated the possible implication of NAT1 in adaptive immune responses to aromatic hydrazines. Moreover, significant activity of NAT1 was found in dendritic cells, T cells and keratinocytes.
Lori Millner from Professor David Hein's laboratory presented differences in arylamine-induced mutagenesis by using alternative mRNA isoforms of human NAT1 (NATa and NATb transcripts) suggesting that following exposures to arylamines, cancer risk may be modulated in tissues expressing both NATa and NATb transcripts.

Dr Julien Dairou (Paris Diderot-Paris 7 University) reported that uncoupling between the first and the second step of the NAT catalytic reaction occurs with human NAT1. For instance, in the presence of folate alone or certain folate-related molecules, NAT1 but not NAT2 behaves as an acetyl-CoA hydrolase.

Dr Jutta Bonifas from Professor Brunhilde Blömekes laboratory talked about keratinocyte differentiation and human NAT1 expression. She demonstrated that NAT1 activity in human keratinocytes varies according to the cell cycle-phase distribution.

Dr Florent Busi (Paris Diderot-Paris 7 University) reported that cadmium alters the biotransformation of carcinogenic arylamines by NAT enzymes in vitro and in vivo [2].

\section{Structures \& enzymatic functions of NAT}

Professor Carston Wagner (University of Minnesota, MN, USA) began this session by demonstrating the covalent inhibition of human and hamster NATs by arylnitroso metabolites. Human NAT1 was found to be more sensitive than NAT2 to these compounds.

Professor Fernando Rodrigues-Lima (Paris Diderot-Paris 7 University) reported the 3D structure of the Bacillus anthracis NATC isoform. Surprisingly, this NAT was found to possess the 'eukaryote-specific 17-residues insertion' and to possess a specific mode of acetyl-CoA recognition.

Nicola Laurieri (Oxford University, Oxford, UK) reported the synthesis and the characterization of a selective human NAT1 and mouse NAT2 inhibitor which displayed a distinctive color change upon binding to these isoforms but not to other NATs.

Elodie Sanfins (Paris Diderot-Paris 7 University) reported that human NAT1 was inhibited by $13-\mathrm{nm}$ carbon black 
nanoparticles owing to irreversible binding and subsequent secondary structure alterations of the protein. Cellular studies indicated NAT-dependent metabolism could be modulated by exposure to these nanoparticles [3].

\section{NAT in microorganisms}

Professor Edith Sim (Oxford University) described a novel reductase enzyme present in the NAT operon of Mycobacterium smegmatis. Characterization of the gene product confirmed that it had reductase activity but not towards dihydrofolate as previously noted.

Xavier Kubiak (Paris Diderot University) presented the identification and characterization of NAT variants from three different clinical strains of Legionella pneumophila. These variants have an unusual length of 322 amino acids and possess up to 16 sequence variations that impact their substrate preferences.

Areej M Abuhammad (Oxford University) described the crystal structure of Mycobacterium marinum NAT in complex with hydralazine and proposed a novel mechanism of acetylation, whereby an acetyl group is transferred from acetyl coenzyme A to the heterocyclic aromatic $\mathrm{N}$ atom of hydralazine.

The last talk was given by Professor Jean-Marie Dupret (Paris Diderot-Paris 7 University), who presented the potential use of fungal NAT enzymes for bioremediation of aromatic amine pollutants in soil [4].

In conclusion, the presentations generated lively discussions and a number of articles covering the data presented in the workshop have been published since the meeting.

\section{Acknowledgements}

The meeting was sponsored by Paris Diderot University, Société de Pharmaco-Toxicologie Cellulaire, Roche (Neuilly/Seine, France), Promega (Charbonniere les Bains, France) Biorad (Marne la Coquette, France) Sigma-Aldrich (Saint-Quentin
Fallavier, France), Fermentas (Villebon sur Yvette, France), Beckman-Coulter (Roissy, France) Dominique Dutscher (Brumath, France) and Invitrogen (Cergy, France).

\section{Financial \& competing interests disclosure}

The authors have no relevant affiliations or financial involvement with any organization or entity with a financial interest in or financial conflict with the subject matter or materials discussed in the manuscript. This includes employment, consultancies, honoraria, stock ownership or options, expert testimony, grants or patents received or pending, or royalties.

No writing assistance was utilized in the production of this manuscript.

\section{References}

1 Tiang JM, Butcher NJ, Cullinane C et al. RNAi-mediated knock-down of arylamine $N$-acetyltransferase-1 expression induces E-cadherin up-regulation and cell-cell contact growth inhibition. PLoS ONE 9(6), e17031 (2011)

2 Ragunathan N, Dairou J, Sanfins E et al. Cadmium alters the biotransformation of carcinogenic aromatic amines by arylamine $N$-acetyltransferase xenobiotic-metabolizing enzymes: molecular, cellular, and in vivo studies. Environ. Health Perspect. 118(12), 1685-1691 (2010).

3 Sanfins E, Dairou J, Hussain S et al. Carbon black nanoparticles impair acetylation of aromatic amine carcinogens through inactivation of arylamine $\mathrm{N}$-acetyltransferase enzymes. ACS Nano. 5(6), 4504-4511 (2011).

4 Silar P, Dairou J, Cocaign A et al. Fungi as a promising tool for bioremediation of soils contaminated with aromatic amines, a major class of pollutants. Nat. Rev. Microbiol. 9(6), 477 (2011).

\section{Website}

101 University of Louisville, Department of Pharmacology and Toxicology, Consensus Human Arylamine $N$-Acetyltransferase Gene Nomenclature http://louisville.edu/medschool/ pharmacology/consensus-human-arylaminen-acetyltransferase-gene-nomenclature 